\title{
ARKCoS: artifact-suppressed accelerated radial kernel convolution on the sphere
}

\author{
F. Elsner ${ }^{1}$ and B. D. Wandelt ${ }^{1,2}$ \\ ${ }^{1}$ Institut d'Astrophysique de Paris, UMR 7095, CNRS - Université Pierre et Marie Curie (Univ Paris 06), 98 bis blvd Arago, \\ 75014 Paris, France \\ e-mail: elsner@iap.fr \\ 2 Departments of Physics and Astronomy, University of Illinois at Urbana-Champaign, Urbana, IL 61801, USA \\ Received 25 March 2011 / Accepted 7 June 2011
}

\begin{abstract}
We describe a hybrid Fourier/direct space convolution algorithm for compact radial (azimuthally symmetric) kernels on the sphere. For high resolution maps covering a large fraction of the sky, our implementation takes advantage of the inexpensive massive parallelism afforded by consumer graphics processing units (GPUs). Its applications include modeling of instrumental beam shapes in terms of compact kernels, computation of fine-scale wavelet transformations, and optimal filtering for the detection of point sources. Our algorithm works for any pixelization where pixels are grouped into isolatitude rings. Even for kernels that are not bandwidth-limited, ringing features are completely absent on an ECP grid. We demonstrate that they can be highly suppressed on the popular HEALPix pixelization, for which we develop a freely available implementation of the algorithm. As an example application, we show that running on a high-end consumer graphics card our method speeds up beam convolution for simulations of a characteristic Planck high frequency instrument channel by two orders of magnitude compared to the commonly used HEALPix implementation on one CPU core, while typically maintaining a fractional RMS accuracy of about 1 part in $10^{5}$.
\end{abstract}

Key words. methods: data analysis - methods: numerical - techniques: image processing - cosmic background radiation

\section{Motivation and goals}

Convolving with radial (i.e. azimuthally symmetric) kernels is a key step in some of the most frequently used algorithms during the simulation and analysis of cosmological data sets represented on the celestial sphere, such as maps of the cosmic microwave background (CMB).

All current and future $\mathrm{CMB}$ experiments have many $\left(100-10^{4}\right)$ detectors (e.g., the Atacama Cosmology Telescope, Kosowsky 2003, the South Pole Telescope, Ruhl et al. 2004, the proposed CMBPol mission, Baumann et al. 2009, or the Planck satellite, Planck Collaboration et al. 2011). Simulating the signal in these data sets requires very many beam smoothing operations since each detector map will contain the same CMB map smoothed with a separate beam shape. The same is true for map-making methods that compute the optimal combination of a large number of detectors in an iterative process and therefore also require a huge number of beam smoothing operations (e.g., Tegmark 1997; Natoli et al. 2001; Stompor et al. 2002).

Several CMB analysis techniques, such as a wavelet analysis (e.g., Hobson et al. 1999; Martínez-González et al. 2002; Vielva et al. 2004), and the filtering to detect point sources (e.g., Tegmark \& de Oliveira-Costa 1998; Cayón et al. 2000; González-Nuevo et al. 2006) require smoothing of high resolution maps with symmetric kernels that have (or are wellapproximated as having) compact support on the sphere. In a wavelet analysis, the computational time for the wavelet transform is dominated by the computation of the fine-scale wavelet coefficients. By construction, the fine-scale wavelets are compact in pixel space.

Current practice in CMB data analysis is the near-exclusive use of the fast spherical harmonic transform (FSHT) for convolution with radial kernels (Muciaccia et al. 1997). Mature and highly efficient implementations of this algorithm are publicly available in several packages, such as, e.g., HEALPix ${ }^{1}$ (Górski et al. 2005), GLESP ${ }^{2}$ (Doroshkevich et al. 2005), ccSHT $^{3}$, or libpsht ${ }^{4}$ (Reinecke 2011).

In the vast majority of cases that a spherical transform is calculated during a CMB data analysis, it is to compute a convolution with a radial kernel. Examples are: (1) simulating CMB maps, in which case the radial kernel is the "square root" of the CMB power spectrum; (2) simulating observed detector maps with a symmetric beam profile; (3) filtering to extract point sources, or hot and cold spots on certain scales; and (4) all forms of symmetric wavelet analysis.

While generally correct, this approach is not optimal when convolving high resolution maps with sufficiently compact kernels. We show that significant speed-up is possible with an algorithm that makes nearly optimal use of massively parallel fast Fourier transforms (FFT) on consumer graphical processing units using a hybrid direct-space and Fourier space approach.

The plan of this paper is as follows. We begin with a mathematical definition of the problem and the quantities involved (Sect. 2). We then briefly review the convolution approaches using a direct sum and the FSHT in Sect. 3, while discussing a GPU implementation of the FSHT (Hupca et al. 2010). We introduce our algorithm and its implementation on a consumer GPU in Sect. 4, which also contains benchmark results and tests of the

\footnotetext{
${ }^{1}$ http://healpix.jpl.nasa.gov

2 http://www.glesp.nbi.dk

${ }^{3}$ http://crd.lbl.gov/ cmc/ccSHTlib/doc

${ }^{4}$ http://sourceforge.net/projects/libpsht
} 
numerical accuracy of the algorithm. Finally, we summarize our findings in Sect. 5.

The benchmarks of the algorithms reported in this paper were performed on an Intel Core2 Quad CPU with $2.8 \mathrm{GHz}$ and $8 \mathrm{~GB}$ of random access memory (RAM). The system cost (other than the GPU) was about US\$ 1000. As a reference, we use the popular HEALPix Fortran package version 2.15 and the highly optimized libpsht C++ FSHT library. We note that starting with the latest release, version 2.20, the libpsht routines will also be called by default in the HEALPix package. Our GPU code was timed on a NVIDIA GeForce GTX 480 that we bought for US\$ 500.

\section{Definitions and notation}

It is useful to state the "platonic ideal" of what is to be accomplished. Given a rough map $r$, we would like to calculate the smooth map $s$

$s\left(\hat{n}_{1}\right)=\int_{S^{2}} K\left(\hat{n}_{1}, \hat{n}_{2}\right) r\left(\hat{n}_{2}\right) d^{2} \hat{n}_{2}$,

where $\hat{n}$ denotes a unit vector on the sphere. For a symmetric (or radial) kernel, $K\left(\hat{n}_{1}, \hat{n}_{2}\right)=K\left(\hat{n}_{1} . \hat{n}_{2}\right)$. We introduce the shorthand notation $p . q \equiv \hat{n}_{p} \cdot \hat{n}_{q}=\cos \left(\varangle\left(\hat{n}_{p}, \hat{n}_{q}\right)\right)$, so $K\left(\hat{n}_{1}, \hat{n}_{2}\right)=$ $K(1.2)$.

A band-limited function on the sphere can be defined in spherical harmonic space by specifying a set of spherical harmonic coefficients $a_{\ell m}$ for all $\ell$ from zero up to band-limit $\ell_{\max }$. Unless otherwise stated sums are over all non-zero terms. With the Legendre transform convention

$K_{\ell}=2 \pi \int_{-1}^{1} K(z) P_{\ell}(z) \mathrm{d} z$,

the kernel can be expanded in terms of Legendre polynomials as

$K(p . q)=\sum_{\ell} \frac{2 \ell+1}{4 \pi} K_{\ell} P_{\ell}(p . q)$.

We assume that the kernel has the same band-limit as the input map. Recalling the addition theorem for spherical harmonics $Y_{\ell m p} \equiv Y_{\ell m}\left(\hat{n}_{p}\right)$

$\sum_{m} Y_{\ell m p} Y_{\ell m q}^{*}=\frac{2 \ell+1}{4 \pi} P_{\ell}(p . q)$,

we obtain

$s_{\ell m}=K_{\ell} r_{\ell m}$.

This equation is exact if the $r_{\ell m}$ are known. In many cases of interest, however, the map will be available in a sampled or pixelized representation with a number of pixels $n_{\text {pix }}$. In this case, estimating the $r_{\ell m}$ from the sampled representation may introduce a quadrature error. We keep this in mind when discussing the convolution accuracy in the following.

\section{Methods}

\subsection{Direct sum}

The direct sum follows from the straightforward discretization of Eq. (1)

$s_{p}=\sum_{q} K(p . q) r_{q}$.
It is easy to check that this approach will yield the same output map as Eq. (5) if the $r_{\ell m}$ are calculated by direct sum over the same equal-area pixelization and both map and radial kernel are band-limited functions. In general, this method scales as $O\left(n_{\text {pix }}^{2} \sim \ell_{\text {max }}^{4}\right)$, both in terms of memory accesses and in terms of floating point operations (FLOP). The prefactor can be made small by caching $K(z)$, e.g. by interpolating it in $O(1)$ operations from $O\left(\ell_{\max }\right)$ precomputed values. This also reduces the number of accesses to non-cached memory to $O\left(\ell_{\max }^{2}\right)$.

If the kernel is compact such that $K(p . q)=0 \forall p . q<z_{K}$, i.e. for an angle between $p$ and $q$ larger than a threshold $\theta_{K}$, the operation count reduces by a factor $z_{K} / 2$. For sufficiently compact kernels this method would therefore win over other methods with smaller asymptotic time complexity but larger prefactors.

The direct sum has a great degree of parallelism, at least at the level of $O\left(n_{\text {pix }} \sim \ell_{\max }^{2}\right)$ threads, since each output pixel is the result of a dot product of one row of $K$ with $r$. Theoretically, even more parallelization can be achieved by parallelizing the dot product, though care must be taken to avoid race conditions when accumulating the smoothed map in parallel. In practice, care must be taken to keep the number of non-cached memory accesses low since the computation would otherwise be limited by memory bandwidth. Since the number of memory accesses is of the same order as the number of calculations, the potential for GPU implementation is limited. Direct pixel space convolution will therefore be superior only for kernels that are too small (of widths narrower than a very small number of pixels) to be of broad practical interest.

\subsection{Fast spherical harmonic transform}

Pixelizations consisting of uniformly sampled isolatitude rings allow for a FSHT, with overall scaling $O\left(\ell_{\max }^{3}\right)$ to take advantage of Eq. (5).

In detail, FFTs on the $O\left(n_{\theta} \sim \ell_{\max }\right)$ isolatitude rings (each containing $O\left(n_{\phi} \sim \ell_{\max }\right)$ pixels $)$ are done in $O\left(\ell_{\max }^{2} \log \ell_{\max }\right)$ operations. The resulting Fourier components $b_{m}(\theta)$ can be transformed into spherical harmonic coefficients $r_{\ell m}$ by applying an associated Legendre transform taking $O\left(\ell_{\max }^{2}\right)$ operations per ring, for a total of $O\left(n_{\theta} \ell_{\max }^{2} \sim \ell_{\max }^{3}\right)$ operations. One obtains the smoothed map by multiplying the $r_{\ell m}$ with the kernel coefficients $K_{l}$ and applying the inverse spherical harmonic transform that inverts the above steps in reverse order again taking $O\left(\ell_{\max }^{3}\right)$ operations.

The Legendre transforms therefore dominate the scaling since applying Eq. (5) takes only $O\left(\ell_{\max }^{2}\right)$ time. Furthermore, at high $\ell$, the recursions necessary to compute the associated Legendre functions become increasingly less accurate and need to be done in double precision with frequent rescaling to avoid floating point underflows (Górski et al. 2005). Implementing the FSHT algorithm on consumer GPUs with reduced double precision performance is therefore non-trivial. The inverse spherical harmonic transform was implemented and benchmarked citing $O(10)$ speed gains with respect to the S2HAT ${ }^{5}$ CPU implementation (Hupca et al. 2010).

The FSHT algorithm is popular since it reduces the computational scaling by a factor $\ell_{\max }$ compared to the direct sum and yet yields the same result (for infinite precision arithmetic). Approximating the continuous spherical harmonic transform by a finite, discrete sum over pixels introduces the same error as approximating the kernel integral by such a discrete sum. If a quadrature rule is applied to improve the accuracy of the $r_{\ell m}$,

5 http://www.apc.univ-paris7.fr/ radek/s2hat.html 
the same quadrature weights can be used in Eq. (6) to reach the identically improved results.

Utilizing the popular HEALPix library on a modern CPU, the serial time for a pair of forward and inverse transforms at Planck resolution $\left(n_{\text {side }}=2048, \ell_{\max }=4096\right)$ is $t \approx 460 \mathrm{~s}$. Since using FSHTs is by far the most common algorithm of choice for symmetric kernel convolutions, this is the reference time for comparisons with other algorithmic approaches.

Methods implementing divide-and-conquer schemes for fast transforms on non-Abelian groups have a smaller asymptotic scaling of CPU time with problem size than the FSHT (Driscoll \& Healy 1994; Wiaux et al. 2007). However, these more sophisticated methods require large amounts of RAM to store precomputed information that renders them impractical for problem sizes of interest for CMB maps, with tens of millions of pixels e.g. from Planck. For smaller problem sizes, the comparatively large complexity of the algorithm causes actual implementations to be slower than algorithmically simpler approaches.

\section{Hybrid method}

We now outline a straightforward hybrid method that combines aspects of the direct summation and spherical harmonic transformation approach. It is based on the simple idea of convolving along isolatitude rings via computationally inexpensive FFTs by means of the convolution theorem, and integrating in the longitudinal direction in pixel space.

This hybrid method is redundant in a way that the product of the kernel image and the input map must be evaluated once on every ring prior to the summation. The computational costs amount to $O\left(\ell_{\max } \log \ell_{\max }\right)$ operations for each FFT on a ring, which must be repeated $O\left(n_{\theta} \sim \ell_{\max }\right)$ times for each of the $O\left(n_{\theta} \sim \ell_{\max }\right)$ rings. In total, the algorithm requires $O\left(\ell_{\max }^{3} \log \ell_{\max }\right)$ operations, formally inferior to the conventional FSHT approach. Unlike that method, however, if the convolution kernel has finite support on only $n_{\text {support }}<n_{\theta}$ rings, the computational complexity decreases linearly, $O\left(n_{\text {support }} \ell_{\max }^{2} \log \ell_{\max }\right)$. It is dominated by FFTs for which highly optimized implementations with a small prefactor exist. Furthermore, the algorithm intrinsically offers an extreme amount of data parallelism, making it in particular suitable for an implementation on GPUs with hundreds of cores.

In practice, the algorithm field-of-application is limited to compact kernels. If a kernel is formally non-zero across the entire sphere, but vanishes sufficiently fast beyond a given angular distance $\alpha_{\text {cut }}$, it can be truncated at that radius without introducing significant errors. For the convolution of an isotropic map with power spectrum $C_{\ell}$, the mean quadratic error introduced by this approximation can be estimated to be

$\sigma^{2}=\sum_{\ell=0}^{\ell_{\max }} \frac{2 \ell+1}{4 \pi} \Delta K_{\ell}^{2} C_{\ell}$,

where $\Delta K_{\ell}$ is the Legendre expansion of the difference of the exact and the truncated kernel.

\subsection{Overview of the algorithm}

We now describe the GPU implementation of the convolution algorithm for an input map in HEALPix format in greater detail. We visualize the individual steps of the algorithm in Fig. 1.

HEALPix maps with resolution parameter $n_{\text {side }}$ are divided into three regions, the north polar cap, the equatorial region, and the south polar cap. Each of the two caps consist of $n_{\text {caps }}=$ $n_{\text {side }}-1$ rings, where the $n$th ring (counted from the pole) contains $4 n$ pixels. The equatorial region comprises $n_{\text {equ }}=2 n_{\text {side }}+1$ rings with a fixed number of $4 n_{\text {side }}$ pixels per ring.

We perform a real-to-complex Fourier transform of length $n_{\mathrm{FFT}}$ on each ring, where $n_{\mathrm{FFT}}=4 n_{\text {side }}$ in the equatorial region but only $n_{\mathrm{FFT}}=4 n$ in the polar caps. We then zero-pad the Fourier coefficients around the poles to generate a rectangular array of $4 n_{\text {side }}-1$ sets of $2 n_{\text {side }}+1$ Fourier coefficients each. The rectangular shape of this array allows us to use the batch FFT mode of our FFT library for the Fourier convolutions that gives us a significant time saving since FFTs dominate our computational time budget. As every ring in the polar and every other ring in the equatorial region of a HEALPix map is shifted by $\phi_{0}=\pi /(4 n)$ and $\phi_{0}=\pi /\left(4 n_{\text {side }}\right)$, respectively (Górski et al. 2005), we compensate for this distortion by phase-shifting the $m$ th Fourier coefficient

$b_{\mathrm{m}}^{\prime}(\theta)=b_{\mathrm{m}}(\theta) \mathrm{e}^{\mathrm{i} m \phi_{0}}$.

After preparing the input map in this way, we can start the convolution process, which loops over all rings in the output map. For each ring at latitude $\theta_{0}$, we generate a kernel grid of size $n_{\text {support }} \times 4 n_{\text {side }}$ pixels and place the kernel at its center at $(\theta, \phi)=\left(\theta_{0}, 0\right)$. To finally interpolate the kernel on this grid, we first calculate the angular distance $\alpha$ between a pixel located at $(\theta, \phi)$ and the kernel. A Taylor expansion to second order in $\alpha$ allows us to rewrite the equation in a simplified way

$$
\begin{aligned}
\alpha^{2} \approx 2 & {\left[\sin ^{2}\left(1 / 2\left(\theta+\theta_{0}\right)\right)+\sin ^{2}\left(1 / 2\left(\theta-\theta_{0}\right)\right)\right] } \\
& -2\left[\sin ^{2}\left(1 / 2\left(\theta+\theta_{0}\right)\right)-\sin ^{2}\left(1 / 2\left(\theta-\theta_{0}\right)\right)\right] \cos (\phi) .
\end{aligned}
$$

Using this parametrization of the angular distance has the advantage of making a numerically expensive arccosine operation redundant. Though Eq. (9) is formally only applicable in the small angle regime, we can exactly compensate for the error by systematically biasing the kernel evaluation. To this end, we first define $\epsilon(\alpha) \equiv \cos (\alpha)-\left(1-1 / 2 \alpha^{2}\right)$ as the error introduced by the approximation. Instead of precomputing a table of the form $\left[\alpha^{2}, K(\alpha)\right]$, we then store a datastructure containing the values $\left[\alpha^{2}, K(\alpha+\epsilon(\alpha))\right]$. To evaluate the kernel, we interpolate linearly from the table.

After interpolating the kernel on the grid, we perform an efficient radix-2 batch FFT. The kernel Fourier coefficients are then multiplied by those of the input map. As a next step, for each ring pixel, we calculate the summation over $n_{\text {support }}$ elements in the $\theta$-direction. Finally, we store the output-ring Fourier coefficients and continue to the next ring.

To transform back to a HEALPix map, we reverse phaseshift the rings and perform a complex-to-real backward FFT on all output rings. In polar rings, we truncate the Fourier expansion to the Nyquist frequency of each individual ring. An alternative procedure would have been to explicitly alias the super-Nyquist modes to sub-Nyquist modes for each ring. Our tests show that this produces negligible differences for maps with a band-limit $\ell_{\max } \leq 2 n_{\text {side. }}$

Our approach avoids the recursions for the associated Legendre functions. Therefore, the entire algorithm could in principle be computed in single precision, which renders it particularly suitable for inexpensive consumer GPUs that have limited double precision capability. However, for very narrow or highly varying kernel in particular, calculating the angular distance between a given pixel and the kernel center, Eq. (9), 


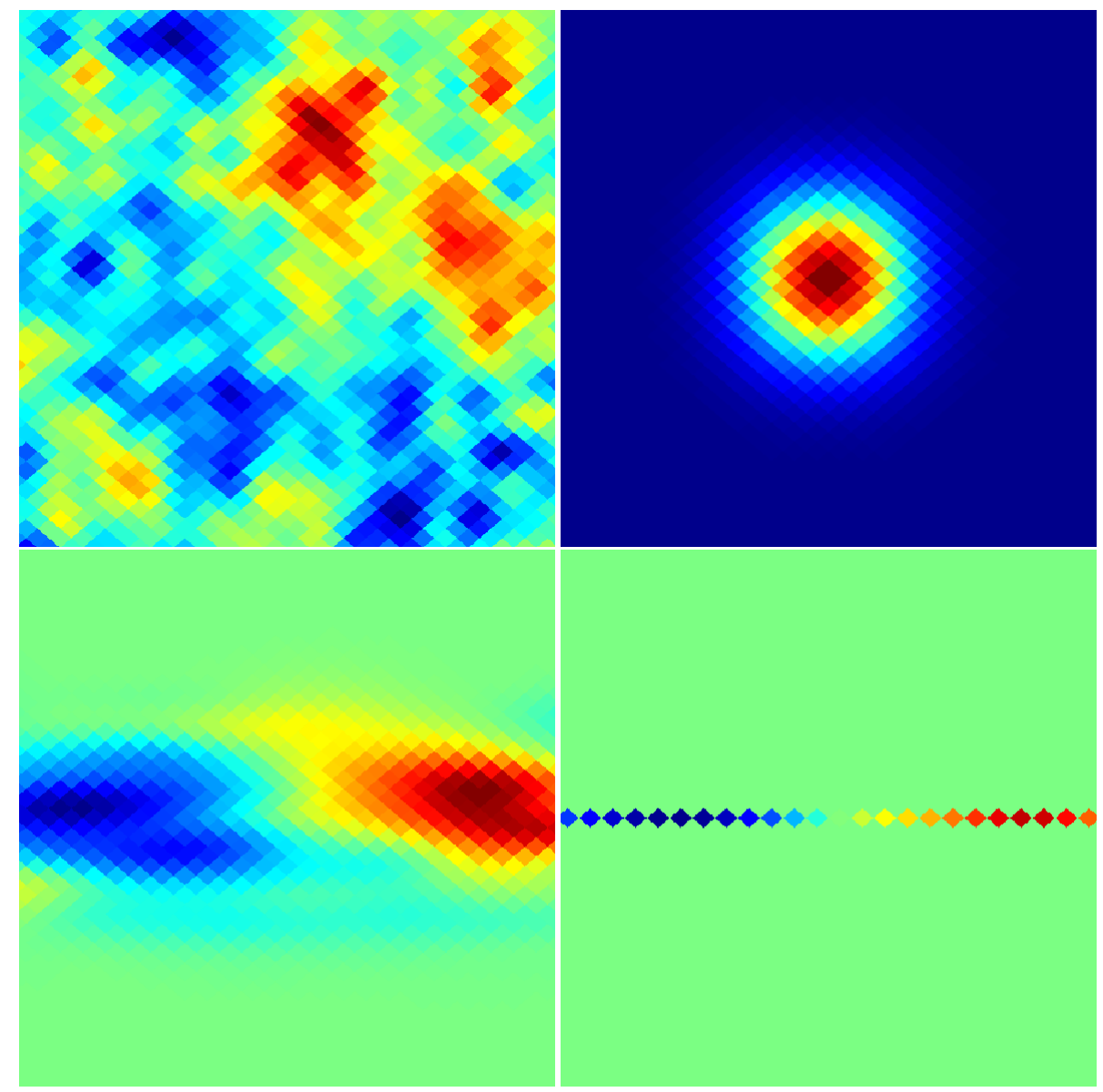

Fig. 1. Illustration of the convolution algorithm. To smooth a specific ring of the input map (upper left), we first interpolate the kernel on the grid, centered on this particular ring (upper right). After a ringwise Fourier transformation of both map and kernel, we multiply the two components (lower left, transformed to pixel space for illustrative purposes). Finally, we perform the summation alongside the longitudinal direction and write the inverse Fourier transformed result to the output (lower right image). becomes imprecise. We therefore compute this quantity using double precision arithmetic for the entire calculation. Further performance improvements at the $10 \%$ level can be realized by partially relaxing this requirement for sufficiently smooth kernels or less stringent accuracy goals.

\subsection{Implementation and benchmarks}

The algorithm was implemented in $\mathrm{C}++$ using the NVIDIA CUDA 3.2 programming toolkit ${ }^{6}$ to generate GPU-related code sequences. We also implemented the hybrid algorithm for the CPU mostly for validation purposes. To obtain a program that runs efficiently on GPUs, a basic understanding of the hardware properties is necessary. We therefore discuss some of the relevant aspects in the following.

GPUs are streaming devices designed for highly parallel high throughput data processing. The hardware used here, a NVIDIA GeForce GTX 480, is a consumer GPU featuring 15 multiprocessors with a total of 480 shader processors. It is equipped with $1.5 \mathrm{~GB}$ of memory and has a nominal peak performance of $1.3 \mathrm{TFLOP} / \mathrm{s}$ in single precision. The value for double precision arithmetic would be half as large but has been intentionally degraded by an additional factor of four by the vendor. For comparison, we note that the performance of the quad-core CPU used for our benchmark tests is about $45 \mathrm{GFLOP} / \mathrm{s}$.

The latency of main memory accesses with a theoretical bandwidth of $177 \mathrm{~GB} / \mathrm{s}$ is large: we typically expect a delay of several hundred clock cycles from the fetch command to the

\footnotetext{
${ }^{6}$ http://developer.nvidia.com/object/ cuda_3_2_downloads.html
}

point where the requested datum is actually available. In the latest generation of NVIDIA GPUs, a L1 cache of up to $48 \mathrm{~KB}$, dedicated to a specific multiprocessor, and a global L2 cache of $768 \mathrm{~KB}$ may reduce the memory latency. Besides main memory, the GPU offers up to $48 \mathrm{~KB}$ of low latency shared and $64 \mathrm{~KB}$ of cached constant memory. In addition, at most 63 registers with virtually instantaneous access and the highest data throughput rate are available per thread.

In general, there are two means of hiding memory latencies: thread-level parallelism and instruction-level parallelism. On GPUs, threads are very lightweight and the switching between them is fast. Common practice is therefore to divide the work load over considerably more threads than physically available computing cores. The second strategy is less obvious. It attempts to calculate several unconditional outputs within the same thread. If a thread encounters a cache miss while computing result $\mathrm{A}$, it can partially hide the latency by continuing to work on the independent result B. Our tests show that reducing the total number of active threads by exploiting instructionlevel parallelism enhances code performance by several tens of percent.

Computations on the GPU are triggered by launching kernels (not to be confused with "convolution kernels"). As a parameter to such a function call, we have to specify how the work load should be processed. More precisely, we define a grid consisting of $1 \mathrm{D}$ or $2 \mathrm{D}$ blocks that are consecutively assigned to a GPU multiprocessor. Each block contains information about the number of threads to be executed on the device.

Our hybrid algorithm can be implemented in a form that is particularly well suited to calculations on GPUs. After transferring the regridded input map to device memory, we use the 
CUFFT library of toolkit version 3.1 to compute a ringwise outof-place real-to-complex FFT on the input map. In contrast to claims in the vendor's release notes accompanying the latest version 3.2, we found the predecessor of the algorithm to be more efficient in terms of both computational performance and memory consumption, though it proves less accurate for certain transformation lengths.

To interpolate the convolution kernel on the grid, we launch a GPU kernel comprising a two-dimensional grid of $n_{\text {side }} / 512 \times$ $n_{\text {support }}$ blocks with 128 threads. Each thread computes the output for 8 different pixels within a ring, with a stride given by the block width. For an efficient interpolation, we stored the longitudes of all rings and the interpolated convolution kernel in constant memory. This code section is compute bound and can be accelerated by taking advantage of the grid symmetries. We calculate the kernel only on the first $2 n_{\text {side }}+1$ pixels of a ring and only for the northern hemisphere.

After the function call has been completed, we execute a real-to-complex batch FFT on the kernel map.

The reduction along the $\theta$-direction is computed partially using thread-save atomic add operations, available on GPUs of compute capability 2.0 and higher. We launch a GPU kernel with a two-dimensional grid of $\left\lceil\left(2 n_{\text {side }}+1\right) / 64\right\rceil \times\left\lceil n_{\text {support }} / 32\right\rceil$ blocks with $64 \times 2$ threads. Each thread accumulates the product of input map and kernel on up to 32 rings in a local variable and adds the result via an atomic operation to the global output map. A block performs this calculation on the northern and southern hemisphere simultaneously. Although a sophisticated design pattern for the common reduction problem exists, we found this approach to be more efficient because we have to deal with non-contiguous memory accesses. This function is memory bound, but we reach up to $145 \mathrm{~GB} / \mathrm{s}$ sustained memory throughput, above $80 \%$ of the theoretically achievable peak performance.

We finally compute an inverse complex-to-real FFT before we transfer the data back to host memory.

In contrast to the exact solution, we find the error in the first and last ring to be unexpectedly enhanced. This is probably the result of amplified numerical errors. Although more of a cosmetic correction than one motivated by accuracy considerations, we recalculate the values of these eight pixels via a direct sum.

With an implementation as described above, a significant speedup compared to a FSHT-based convolution can be realized for compact kernels. We show the results of our benchmark tests in Fig. 2, where we compare the runtime of our algorithm for different map resolution parameters and kernel sizes to that of the HEALPix FSHT, and to the optimized libpsht library on a single CPU core. We note that the GPU timings include the time for transferring the input map from host memory to GPU memory and the output map from the GPU back to the host.

We observe a dependence of the performance gain on the resolution parameter $n_{\text {side }}$. For comparatively low-resolution maps and small-to-moderate kernel sizes, we find the poorer numerical efficiency to be the result of too small a workload to enable the efficient use of the available GPU hardware resources. In addition, we explicitly optimized our code for fast convolutions at $n_{\text {side }}=2048$. For a kernel support of $1^{\circ}$, the runtime is completely dominated by the computational costs of the FFTs. However, with an increasing kernel size, the scaling behavior changes as both the kernel evaluation and the multiplication of the Fourier coefficients of kernel and map become more and more expensive. Accordingly, the higher efficiency that we achieve flattens out towards higher kernel diameters. We specify the fractional computational costs of the different code sections

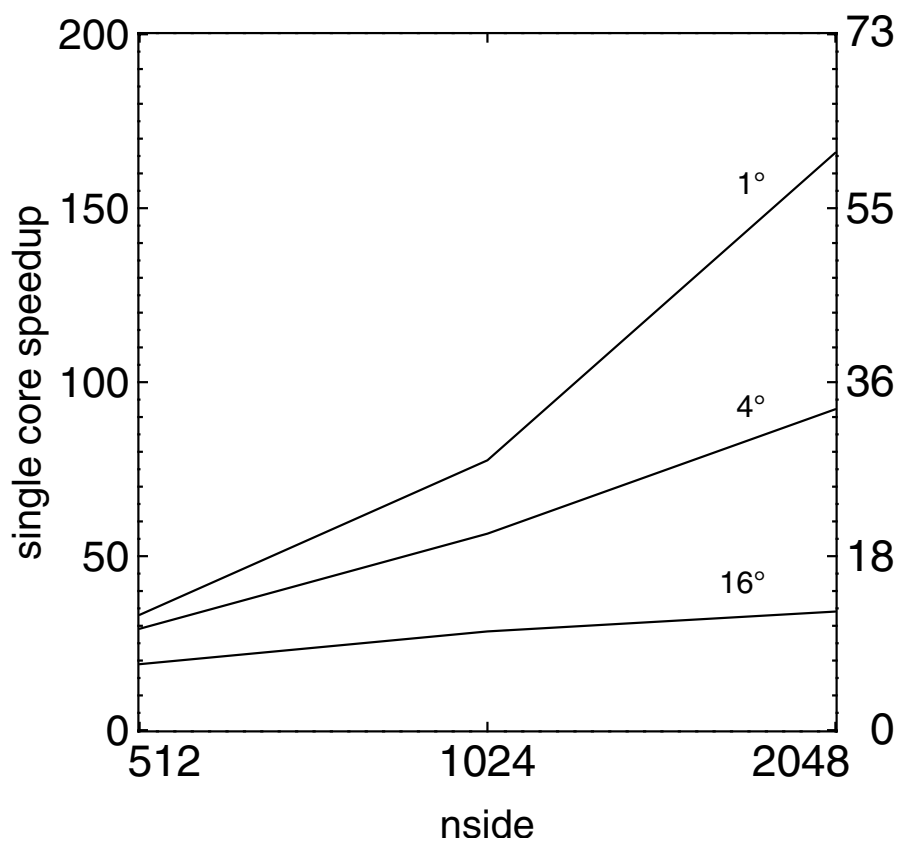

Fig. 2. Performance gain of the GPU-based convolution code when compared to the HEALPix FSHT-based implementation (left axis) and the libpsht library (right axis) running on a single CPU core for different map resolution parameters and kernel support.

Table 1. Breakdown of the total runtime into the contributions of the three most important code sections for the convolution of a map at $n_{\text {side }}=2048$ with kernels of various sizes.

\begin{tabular}{ccrr}
\hline \hline Kernel support & $1^{\circ}$ & $4^{\circ}$ & $16^{\circ}$ \\
\hline FFTs & $57 \%$ & $47 \%$ & $39 \%$ \\
Kernel evaluation & $19 \%$ & $31 \%$ & $39 \%$ \\
Ring reduction & $10 \%$ & $16 \%$ & $21 \%$ \\
Others & $14 \%$ & $6 \%$ & $1 \%$ \\
\hline
\end{tabular}

for the convolution at $n_{\text {side }}=2048$ in Table 1 . Since the three major parts of the algorithm take up comparable amounts of GPU time, further implementation optimizations for the GPU are unlikely to result in performance gains significantly larger than the $10 \%$ level.

In the case of the narrow convolution kernels often encountered during CMB map beam-convolution processes, performance improvements of up to two orders of magnitudes can be achieved. For example, smoothing a HEALPix map with resolution $n_{\text {side }}=2048, \ell_{\max }=4096$ using a Gaussian kernel of 4.7' full width at half maximum $(F W H M)$, a realistic value for the Planck 217 GHz channel (Planck HFI Core Team et al. 2011) takes about $t_{\mathrm{ARKCOS}}=2.2 \mathrm{~s}$ on the GPU, whereas the FSHTbased approach requires $t_{\mathrm{HEALPix}}=460 \mathrm{~s}$ and $t_{\text {libpsht }}=160 \mathrm{~s}$ on one CPU core. Although the intrinsically parallel structure of the algorithm can be most beneficially exploited when run on GPUs, a CPU-based implementation may also be appropriate for very compact kernels. For the setup discussed above, the convolution takes about $t_{\mathrm{ARKCoS}}^{\mathrm{CPU}}=20 \mathrm{~s}$ on one single CPU core, which is still considerably faster than the FSHT-based code.

The cost of realizing these performance gains is to add a GPU at about half of the cost of the quad-core host system. To compare performance per hardware dollar, the GPU timings should be compared to half the CPU timings. 

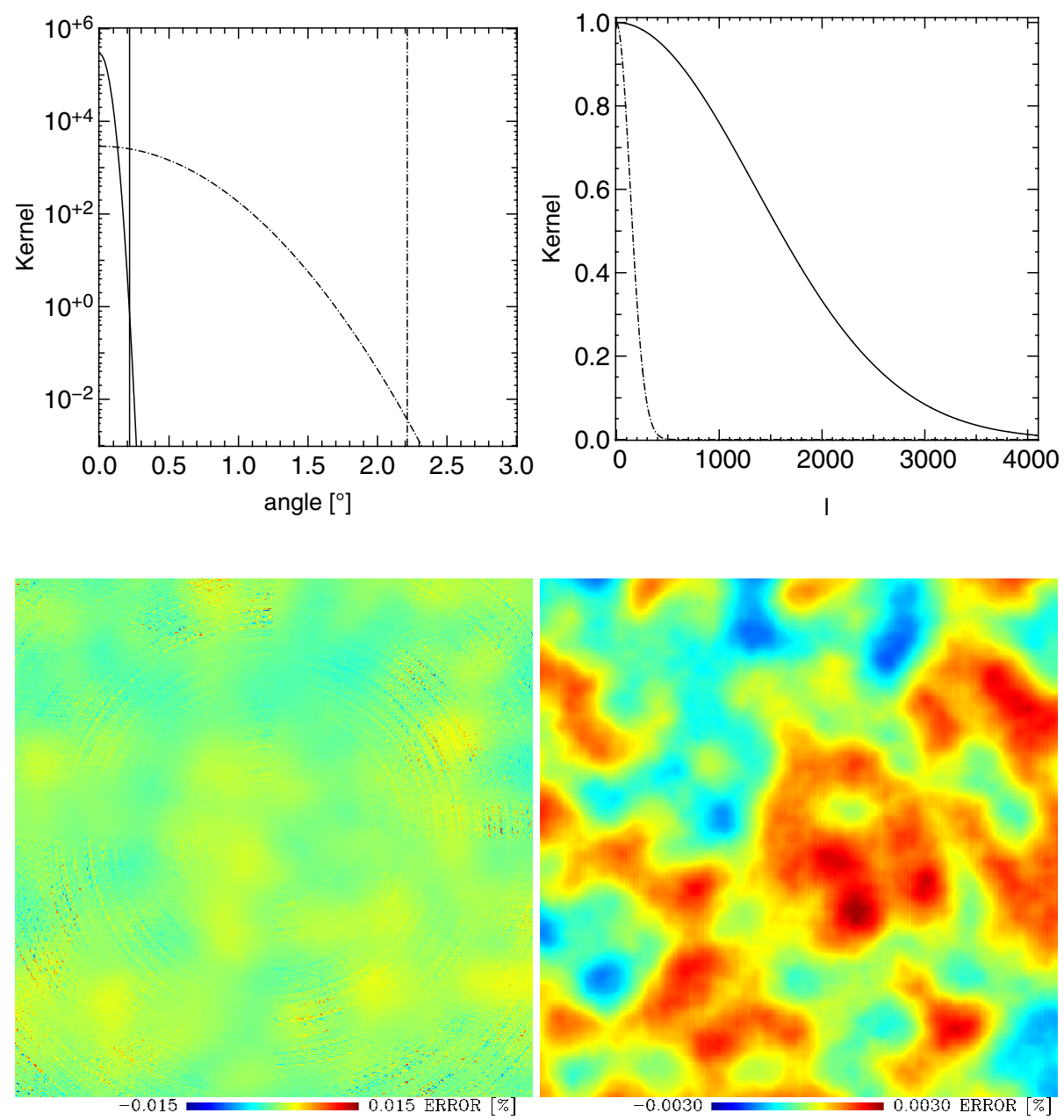

Fig. 3. Profile of the kernels used for our accuracy tests. Left panel: we show the pixel space representation of a Gaussian kernel with $6^{\prime}$ (solid line) and $1^{\circ}$ FWHM (dashed line). The vertical lines indicate the angle beyond which the kernel is truncated. Right panel: legendre expansion of the kernels (solid line: $6^{\prime} F W H M$, dashed line: $1^{\circ} F W H M$ ). Kernels that are compact in pixel space cover a wide range of modes in spherical harmonic space and vice versa.

Fig. 4. Result of the accuracy test. The difference between a map smoothed in spherical harmonic space and a map smoothed with our hybrid method is at most $1.5 \times 10^{-4}$ in a small number of outliers around the north pole (left panel). These are generated by numerical errors in the CUFFT library v. 3.1 for specific HEALPix ring lengths and are absent when using the slightly slower CUFFT v. 3.2. The larger scale $O\left(10^{-5}\right)$ error both at the pole and in the equatorial region (right panel) is caused by small inaccuracies in the kernel evaluation. In this test, a Gaussian kernel with $1^{\circ} \mathrm{FWHM}$ was used. Each patch is $10^{\circ}$ on the side.

\subsection{Accuracy tests}

Our accuracy goal was to achieve a fractional root mean square (RMS) accuracy of $O\left(10^{-4}\right)$ or lower, which would be sufficient for most CMB applications. We assessed the accuracy of the newly developed algorithm on the basis of both the pixel space representation of the convolved maps, and their power spectra.

For the first test, we computed difference maps of the output generated by ARKCoS and HEALPix. Using a Gaussian kernel with $1^{\circ} F W H M$, as plotted in Fig. 3, we show the result of the comparison in Fig. 4. We note that we normalized the difference using the RMS of the reference map to obtain a relative percentage error. We find the small remaining residual around the polar caps to be dominated by outliers produced by the FFT library for specific transformation lengths ${ }^{7}$, whereas inaccuracies in the kernel evaluation prevail in the equatorial region. Averaged over the entire map, ARKCoS reproduces the results from the HEALPix package for different kernels with a fractional RMS error of at most $O\left(10^{-4}\right)$, which decreases rapidly for kernel sizes $\gtrsim 0.5^{\circ} \mathrm{FWHM}$.

As a second test, we compared the power spectrum of the convolved map with the theoretical expectation. With a FWHM of $6^{\prime}$, we chose a very narrow Gaussian kernel close to the grid resolution at $n_{\text {side }}=2048$ that is no longer band-limited

\footnotetext{
7 Tests with the identical code linked to the more recent CUFFT library version 3.2 made these outliers disappear, but performance suffered at the $15 \%$ level.
}

at $\ell_{\max }=4096$. The reference power spectrum used in this test was calculated exactly from the spherical harmonic representations of input map and kernel. For one realization, the result is shown in the left-hand panel of Fig. 5, and interpreted in terms of the cross-power spectrum between the map and the induced error.

In addition, we show the power spectrum of the difference map, where we again compare the output of our algorithm to the exact solution. Here, the reference map was derived via Eq. (5) from the spherical harmonic coefficients of input map and kernel. For one realization, we show the result in the right-hand panel of Fig. 5, it represents the auto power spectrum of the error. In both cases, we find the error in the power spectra to be subdominant over the full dynamical range of about $14 \mathrm{mag}^{8}$, showing that the algorithm does not introduce a significant level of artificial mode coupling.

We conclude with the remark that highly compact kernels of scale-lengths smaller than $\approx 10^{\prime} F W H M$, a regime of particular relevance to beam convolution at the resolution of the Planck high frequency instrument, will suffer from truncation errors if a band-limit of $\ell_{\max }=4096$ is imposed. As a result, the backtransformed pixel space representation starts to show ringing artifacts. In contrast to FSHT-based algorithms, it is possible to suppress this effect in our convolution scheme. The first modification of the algorithm concerns the treatment of super-Nyquist

\footnotetext{
8 Note that in Fig. 5, we show the power spectra multiplied by $\ell(\ell+$ $1) / 2 \pi$. This factor has to be taken into account when the dynamical range of the simulation is to be assessed.
} 
F. Elsner and B. D. Wandelt: ARKCoS: Accelerated radial kernel convolution on the sphere
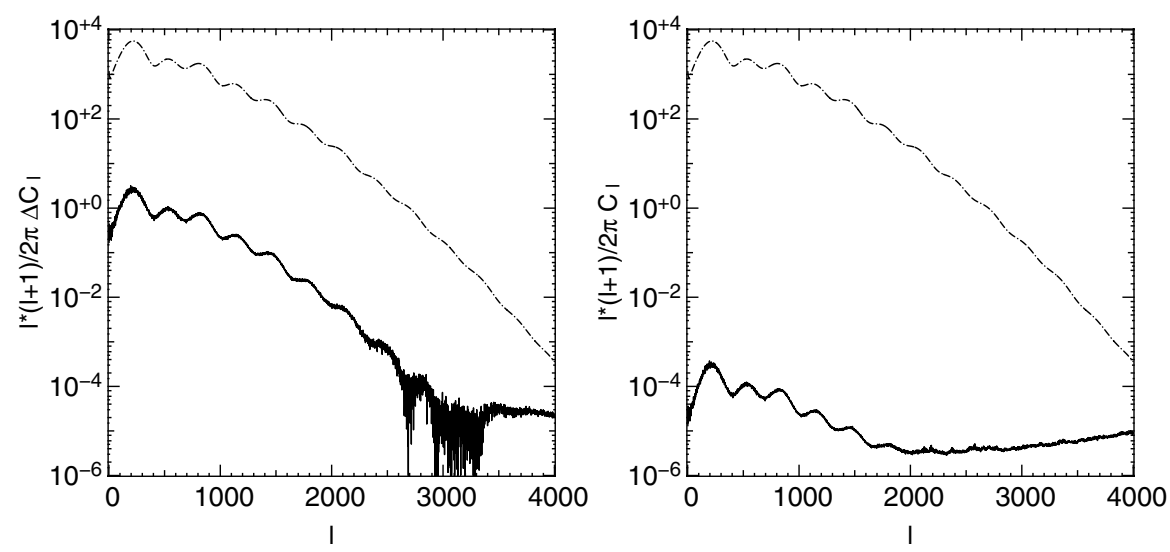

Fig. 5. Power spectrum accuracy. Left panel: for one particular realization, we plot the difference between the power spectrum of a map convolved with a narrow Gaussian, FWHM $=6^{\prime}$ and the power spectrum of the exact convolution. Right panel: we show the power spectrum of the difference map, computed from the convolution of a map using ARKCoS and the exact solution. For comparison, we also show the expected power spectrum of the exact convolution in both panels (dashed lines). Note that since we are comparing to the exact convolved power spectrum this error measure includes the HEALPix quadrature error.
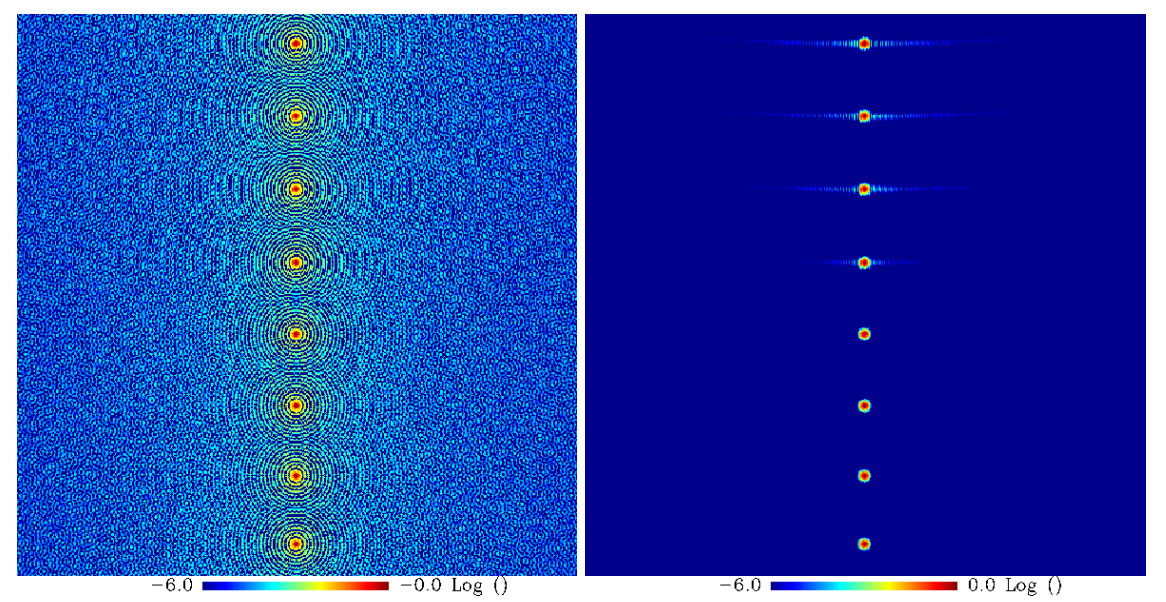

Fig. 6. Reduced ringing-artifacts in our enhanced hybrid algorithm. Left panel: the convolution of point sources with a Gaussian kernel of 4.7' FWHM using a FSHT-based algorithm at $n_{\text {side }}=2048$ causes extended residuals. Right panel: the result obtained with ARKCoS only shows a suppressed ringing pattern in the longitudinal direction in the polar caps (upper four point sources). In the equatorial region, the artifacts cancel out exactly (lower four point sources). Each patch is $13^{\circ}$ on the side, the logarithmic color scale counts representing factors of 10 from the maximum. For ARKCoS, the ringing patterns are too small to be visible on a linear scale.

modes in the polar caps. These modes are available to us because we supersample the kernel in direct space on $4 n_{\text {side }}$ points on all rings. After performing the forward Fourier transform of the input map, we now duplicate the coefficients to obtain a fully populated rectangular grid with $2 n_{\text {side }}+1$ elements on all rings. Likewise, we add the super-Nyquist modes to the sub-Nyquist modes prior to calculate the inverse Fourier transform of the output map. This (optional) step in the algorithm adds a factor of less than two to the computational time. In the equatorial region, the error can be removed completely if we slightly alter the algorithm on every unshifted ring. Here, we start the convolution using the unmodified Fourier transform of the input map, that is, we do not apply Eq. (8). We instead take into account the offset on every other ring during the kernel evaluation, i.e., we substitute $\cos (\phi)$ with $\cos \left(\phi-\phi_{0}\right)$ in Eq. (9). In Fig. 6, we compare the output of our modified algorithm to that of a FSHT-based scheme for the convolution of several point sources with a Gaussian beam of width $4.7^{\prime} F W H M$ at $n_{\text {side }}=2048$. The conventional approach suffers from spurious ringing effects that extend well beyond the formal support of the kernel. Using ARKCoS, the artifacts are completely absent in the equatorial region, and suppressed and confined to the latitudinal direction in the polar caps. We note that on the more regular ECP-grid, the ringing pattern would vanish exactly on the entire sphere without the need to modify the algorithm.

\section{Discussion and conclusion}

We have presented an implementation of a GPU-accelerated hybrid algorithm for radial kernel convolution on the sphere.
It performs the convolution along isolatitude rings in Fourier space and integrates in longitudinal direction in pixel space. We call this algorithm ARKCoS. As the computational costs scale linearly with the kernel support, the method is most beneficial for convolution with compact kernels. Typical applications include CMB beam smoothing, symmetric wavelet analyses, and point-source filtering operations.

For a convolution with compact kernels, we find that our implementation realizes real performance gains of up to $5000 \%$, depending on the problem size, for a $50 \%$ increase in system cost relative to the most widely used FSHT implementation in the HEALPix library running in parallel on a quad-core CPU. When compared to the more finely tuned libpsht FSHT library, again running on four cores, we still find significant performance gains, up to $1800 \%$.

We assessed the numerical accuracy of the algorithm by comparing the convolved output map to the result generated using HEALPix. The outcome typically agrees with the FSHT-based convolution to 1 part in $10^{4}$. Comparing the power spectrum of the output map to the exact solution for a narrow convolution kernel, we find a relative error of smaller than $10^{-3}$. For kernels that are not band-limited, the convolution with a FSHT-based scheme induces ringing artifacts. Using instead a slightly modified implementation of ARKCoS, however, we have demonstrated that a huge reduction in the spurious contribution is possible.

The massively parallel hybrid approach we have presented here is particularly advantageous for convolutions with compact kernels (with support less than $\sim 15^{\circ}$ ) at high resolution ( $n_{\text {side }}=512$ or higher $)$. The GPU we used for our tests has 
1.5 GB of RAM. This is too small to store simultaneously the input and output HEALPix maps at resolution $n_{\text {side }}=4096$ or higher. Possible solutions for future work involve calculating the contribution to the output map from subsets of rings in the input map, either sequentially or in parallel if more than one GPU is available in the same system.

This work deals with radial kernel convolution. We note in closing that there is considerable interest in the algorithmically more difficult problem of asymmetric kernel convolution (Wandelt \& Górski 2001; Wiaux et al. 2005; ASYMFAST, Tristram et al. 2004; FICSBell, Hivon \& Ponthieu, in prep.; FEBECoP, Mitra et al. 2011) either to model the physical optics of CMB experiments more faithfully (Mennella et al. 2011; Planck HFI Core Team et al. 2011) or to detect signals that have locally anisotropic signatures. Having found in this work that our hybrid algorithm vastly accelerates radial kernel convolution, it is easy to imagine generalizations that accelerate asymmetric kernel convolution in a similar way. The ASYMFAST approach (Tristram et al. 2004) reduces the problem of asymmetric beam convolution to $O(10)$ symmetric convolutions. Coupled to our GPU accelerated approach, the convolution with even complex asymmetric kernels and compact support takes less time than the convolution with a single symmetric kernel on a CPU system using FSHTs.

Acknowledgements. We thank the anonymous referee for the comments which helped to improve the presentation of our results. We are grateful to S. Kruger for some early exploratory GPU work. We thank F. Bouchet, C. Lawrence, and S. Gratton for their encouragement at various stages during the project, and E. Hivon for comments on the draft of this paper. BDW was supported by NASA/JPL subcontract 1413479, and NSF grants AST 07-08849, AST 0908693 ARRA and AST 09-08902 during this work. This research was supported in part by the National Science Foundation through TeraGrid resources provided by NCSA under grant number TG-AST100029. Some of the results in this paper have been derived using the HEALPix (Górski et al. 2005), the libpsht (Reinecke 2011), and the FFTW (Frigo \& Johnson 2005) packages.

\section{References}

Baumann, D., Jackson, M. G., Adshead, P., et al. 2009, AIP Conf. Proc., 1141, 10

Cayón, L., Sanz, J. L., Barreiro, R. B., et al. 2000, MNRAS, 315, 757

Doroshkevich, A. G., Naselsky, P. D., Verkhodanov, O. V., et al. 2005, Int. J. Mod. Phys. D, 14, 275

Driscoll, J. R., \& Healy, D. M. 1994, Adv. Appl. Math., 15, 202

Frigo, M., \& Johnson, S. G. 2005, Proc. IEEE, 93, 216

González-Nuevo, J., Argüeso, F., López-Caniego, M., et al. 2006, MNRAS, 369, 1603

Górski, K. M., Hivon, E., Banday, A. J., et al. 2005, ApJ, 622, 759

Hobson, M. P., Jones, A. W., \& Lasenby, A. N. 1999, MNRAS, 309, 125

Hupca, I. O., Falcou, J., Grigori, L., \& Stompor, R. 2010, [arXiv: 1010. 1260]

Kosowsky, A. 2003, New A Rev., 47, 939

Martínez-González, E., Gallegos, J. E., Argüeso, F., Cayón, L., \& Sanz, J. L. 2002, MNRAS, 336, 22

Mennella, A., Bersanelli, M., Butler, R. C., et al. 2011, A\&A, accepted, [arXiv: 1101.2038]

Mitra, S., Rocha, G., Górski, K. M., et al. 2011, ApJS, 193, 5

Muciaccia, P. F., Natoli, P., \& Vittorio, N. 1997, ApJ, 488, L63

Natoli, P., de Gasperis, G., Gheller, C., \& Vittorio, N. 2001, A\&A, 372, 346

Planck Collaboration, Ade, P. A. R., Aghanim, N., et al. 2011, A\&A, accepted, [arXiv: 1101.2022]

Planck HFI Core Team, Ade, P. A. R., Aghanim, N., et al. 2011, A\&A, accepted, [arXiv: 1101.2048]

Reinecke, M. 2011, A\&A, 526, A108

Ruhl, J., Ade, P. A. R., Carlstrom, J. E., et al. 2004, SPIE Conf. Ser. 5498, ed. C. M. Bradford, P. A. R. Ade, J. E. Aguirre, J. J. Bock, M. Dragovan, L. Duband, L. Earle, J. Glenn, H. Matsuhara, B. J. Naylor, H. T. Nguyen, M. Yun, \& J. Zmuidzinas, 11

Stompor, R., Balbi, A., Borrill, J. D., et al. 2002, Phys. Rev. D, 65, 022003

Tegmark, M. 1997, ApJ, 480, L87

Tegmark, M., \& de Oliveira-Costa, A. 1998, ApJ, 500, L83

Tristram, M., Macías-Pérez, J. F., Renault, C., \& Hamilton, J. 2004, Phys. Rev. D, 69, 123008

Vielva, P., Martínez-González, E., Barreiro, R. B., Sanz, J. L., \& Cayón, L. 2004, ApJ, 609, 22

Wandelt, B. D., \& Górski, K. M. 2001, Phys. Rev. D, 63, 123002

Wiaux, Y., Jacques, L., \& Vandergheynst, P. 2005, AGU Fall Meeting Abstracts, A1201

Wiaux, Y., Jacques, L., \& Vandergheynst, P. 2007, J. Comp. Phys., 226, 2359 